UDC: 811.134.2:811.163.41'373

DOI: https://doi.org/10.18485/hispserb.2019.2.ch26

\author{
Luiza Valožić ${ }^{1}$ \\ Universidad de Belgrado \\ Serbia
}

\title{
LA DIVERSIDAD CULTURAL Y LA PUBLICIDAD: ANÁLISIS COMPARATIVO DE ANUNCIOS DE CERVEZA DE ESPAÑA Y SERBIA
}

\begin{abstract}
Resumen
Los medios de comunicación social y la publicidad tienen una relevancia destacada para la sociedad desde la segunda mitad del siglo XIX, que sigue vigente, quizá más que nunca, en el contexto actual. Tanto los medios de comunicación social como la publicidad han sido objeto de estudio desde una multitud de perspectivas teóricas y metodológicas, y el interés por su análisis continúa. Dada su relevancia e influencia en la sociedad, la publicidad también es un ámbito de estudio de interés para las diferentes disciplinas lingüísticas, sobre todo en cuanto a su potencial de influenciar sobre el uso de la lengua y de ser un canal de transmisión de tendencias vinculadas a la globalización.

En nuestro artículo presentaremos los resultados de un análisis comparativo, desde la perspectiva teórica y metodológica de la sociolingüística, de anuncios de cerveza de España y Serbia. También incluimos un análisis ampliado de nuestra variable social, los anuncios de cerveza de España y Serbia, no estrictamente sociolingüístico, al fijarnos en la imagen de los anuncios con el objetivo de observar más de cerca la representación de los receptores del mensaje publicitario.
\end{abstract}

${ }^{1}$ luiza.valozic@gmail.com 
El objetivo de nuestro análisis comparativo es observar y analizar la diversidad cultural y social presente en los anuncios de los dos países mencionados, así como indicar el potencial didáctico del uso del análisis comparativo de la publicidad en clase de ELE.

Palabras clave: la diversidad cultural, la publicidad, la globalización, el análisis sociolingüístico.

\section{Introducción}

La publicidad y el sociolecto publicitario ${ }^{2}$ siguen ofreciendo hoy en día un amplio universo de posibilidades de estudio desde una multitud de disciplinas, y sobre todo desde los diferentes enfoques teóricos de la lingüística. Desde su constitución como actividad profesional bien definida y con un claro objetivo en la segunda mitad del siglo XIX, la publicidad ha experimentado una evolución constante, y sigue teniendo hoy una gran relevancia tanto en los medios de comunicación denominados tradicionales (televisión, radio, prensa) como en el ámbito de internet. El hecho de ser la principal fuente de ingresos de los medios de comunicación le otorga un innegable poder y le dota a la vez de un carácter ubicuo. No solo nuestro espacio cercano, nuestros teléfonos móviles y ordenadores le sirven de soporte a través de internet, sino, al acudir a cualquier medio de comunicación tradicional, o bien con salir a la calle, nos vemos expuestos a sus mensajes. Todos esos mensajes publicitarios, todo el lenguaje usado en la publicidad, conforman un rico campo de estudio sobre todo para las disciplinas lingüísticas, pues son muestras del uso de la lengua desde el punto de la vista sincrónico.

Aparte de su cualidad como un ámbito que refleja el uso sincrónico de la lengua, la publicidad, en su esencia como actividad profesional, es un elemento importante de la producción, la venta y el consumo, y como tal se ve fuertemente influenciada por la globalización económica, que ha propagado su influencia a otros ámbitos como son el político, el científico, el social, el cultural y el lingüístico. En relación con la globalización económica, se delimitan en términos generales dos procesos de cambio social observables en el contexto actual, uno con una tendencia a uniformar el ámbito financiero, político, cultural y social de los países

\footnotetext{
${ }^{2}$ Para situar la variedad publicitaria seguimos la diferenciación descriptiva de las variedades establecida por F. Gimeno (1990: 39), que se desarrolla en el siguiente apartado.
} 
del mundo, y el otro, donde persiste la aspiración a la diversificación mediante la reivindicación de elementos autóctonos sociales, culturales y lingüísticos. Mientras que la tendencia a la mundialización económica y tecnológica puede provocar situaciones de sustitución lingüística de lenguas minoritarias por la lengua mayoritaria, actualmente, el inglés, las tendencias a la autodefinición social, cultural y lingüística son susceptibles de favorecer la conservación de la diversidad social, cultural y lingüística. Es de particular interés para nuestro análisis comparativo este último aspecto, el de la autodefinición, que comentaremos más adelante.

Con el objetivo de observar la mencionada diversidad presente en anuncios publicitarios, centramos el objetivo de nuestro análisis comparativo sincrónico en anuncios de cerveza de España y Serbia para la televisión e internet con un enfoque teórico y metodológico de la sociolingüística.

\section{Marco teórico}

El estudio de la publicidad y del sociolecto publicitario cuenta con una larga tradición que se inicia poco después de su consolidación como actividad profesional bien definida.

Cabe mencionar que no existe un acuerdo entre los investigadores sobre los inicios y etapas de desarrollo de la publicidad como actividad profesional. En nuestro caso, seguimos los postulados de R. Eguizábal (1998), quien sitúa el nacimiento de la publicidad como actividad profesional a mediados del siglo XIX, que es cuando surgen las primeras agencias de publicidad y nace el concepto de marca.

Es de nuestro particular interés el estudio del sociolecto publicitario desde las diferentes disciplinas lingüísticas, aunque, claro, la publicidad ha sido y es estudiada desde otras muchas disciplinas. Algunas de las perspectivas teóricas y metodológicas que han abordado el estudio de la lengua y el uso del sociolecto publicitario son: la semiótica, la lingüística, la pragmática, el análisis del discurso, la sociolingüística, etc. (Valozic 2015: 17-59).

El concreto contexto de la publicidad de bebidas alcohólicas, en el que centramos nuestro análisis comparativo, también ha sido estudiado desde diversos planteamientos teóricos. R. Durán (2005) realiza un análisis de los anuncio de publicidad de alcohol y tabaco en la prensa española, y se centra principalmente en el análisis de la comunicación encubierta desde el marco teórico de la Teoría de la relevancia. M. Ramos y M. ${ }^{a}$ del Mar Rubio (2011) presentan un análisis semiótico de los estereotipos presentes en 
anuncios de cerveza de España, y una de las conclusiones de su estudio es que: "los anuncios de bebidas alcohólicas, más allá del interés comercial que todo acto publicitario supone, reflejan los rasgos de la identidad cultural de una determinada región." (Ramos \& Rubio 2011: 228).

Desde el punto de vista educativo F. Racionero y M. ${ }^{a}$ Ángeles Olivares (2012) presentan un estudio de estereotipos femeninos en los anuncios de España y Brasil, con el objetivo de formar a alumnos críticos en relación con los mensajes publicitarios. Destacaríamos este enfoque, ya que la realización de nuestro estudio ha sido motivada en parte por la aplicación de la publicidad, y sobre todo del análisis comparativo de la publicidad de diferentes países, en clase de ELE. También cabe destacar que muchos libros de texto de ELE incluyen la publicidad, o bien a través de un tema específico, o bien a través de diversos ejercicios.

Además de los estudios mencionados, se puede observar en internet una presencia significativa de tesis y de artículos que tratan el tema de la publicidad del alcohol en general, y de la publicidad de cerveza en particular, así como la presencia de estereotipos y características sociales y culturales en dichos anuncios.

Como hemos comentado al principio, nuestro análisis comparativo tiene un enfoque sincrónico y se fundamenta en el marco teórico y metodológico de la sociolingüística ${ }^{3}$.

El sociolecto publicitario que analizamos en este trabajo describe una doble vertiente, ya que, por un lado, está condicionado por el contexto social, cultural y lingüístico en el que se desarrolla, y, por el otro, influye en el registro oral de la población. La diferenciación descriptiva de las variedades que establece F. Gimeno (1990: 39) nos ofrece un marco claro para situar la variedad publicitaria. El autor especifica las variedades geográficas, sociales y estilos contextuales que refleja la heterogeneidad descriptiva de la lengua. Concretamente, dentro de las variedades sociales, esto es, los sociolectos, se han de diferenciar las variedades generacionales, sexuales, socioeconómicas, de origen y especiales, en función de las variables independientes (edad, sexo, grupo socioeconómico y otras). Dentro de las variedades especiales se distingue el argot, variedades científico-técnicas y variedades sectoriales, dentro de las cuales se sitúa el sociolecto publicitario.

El sociolecto publicitario se caracteriza por la heterogeneidad de los códigos que lo integran, el lingüístico, el paralingüístico (referido

\footnotetext{
${ }^{3}$ Más concretamente, seguimos el planteamiento global e interdisciplinario de la sociolingüística, que engloba dos niveles, uno macrosociolingüístico y uno microsiciolingüístico (Gimeno \& Gimeno 2003: 22-23).
} 
a la tipografía) y el icónico (esto es, las imágenes). Estas características coinciden con la caracterización de M. V. Romero (1993: 9 y sigs.) del sociolecto periodístico. Ambas variedades comparten estos tres códigos, pero su materialización es diferente.

En el caso del sociolecto publicitario, la presencia simultánea de estos tres códigos no es determinante ni excluyente. La presencia del código paralingüístico sí va unida a la presencia del código lingüístico. Esta posibilidad de alternancia de los códigos es otra característica del sociolecto publicitario. Un elemento clave y propio del sociolecto publicitario, que a su vez lo distingue, es la marca o el logotipo de la empresa (Valozic 2015: 76-77).

Las variables sociales independientes de nuestro análisis son anuncios de cerveza para la televisión o internet en formato audiovisual de las marcas de cerveza más populares de España y Serbia, y las variables lingüísticas dependientes son los pronombres personales. Asimismo, incluimos en nuestro trabajo un análisis de la parte de la imagen del sociolecto publicitario, en el sentido de que observaremos los personajes que aparecen en la imagen en relación con las variables lingüísticas, y nos centramos en el criterio de edad y sexo con el objetivo de observar también la dimensión social presente en los anuncios de nuestro corpus a través de estos personajes. Este análisis es adicional y sobrepasaría los límites de un análisis estrictamente sociolingüístico, ya que observamos elementos (personajes de los anuncios) que están dentro de nuestra variable social, que son los anuncios de cerveza de los dos países.

Como hemos mencionado, el sociolecto publicitario está condicionado por el contexto social, cultural y lingüístico, en el que se desarrolla, y aunque no recoge toda la amplitud de dicho contexto, nos permite observar algunas diferencias entre los anuncios de cerveza de España y de Serbia a través del análisis comparativo de las variables de nuestro estudio.

\section{La muestra}

La muestra del estudio la constituyen anuncios en formato audiovisual para la televisión e internet de las marcas de cerveza más populares de España y Serbia ${ }^{4}$. En el caso de España, las marcas de cerveza

\footnotetext{
${ }^{4}$ Los datos correspondientes tanto a las marcas de cerveza más populares de España y de Serbia como de los hábitos de consumo de cerveza en los dos países están extraídos de los sitios web oficiales de las siguientes organizaciones: para España de Cerveceros de España - entidad que agrupa los mayores productores de cerveza de España, y para Serbia
} 
que forman parte de nuestra muestra son: Mahou, Cruzcampo y Estrella Damm, y en el caso de Serbia son: Jelen, Lav у Зајечарско (Zaječarsko) ${ }^{5}$.

La selección de la cerveza como producto para la realización de este análisis comparativo reside en el hecho de que los hábitos de consumo de cerveza son bastante similares en España y en Serbia. La cerveza es una bebida que consume la mayoría de la población adulta, lo que nos permite establecer ciertos paralelismos y observar las posibles diferencias entre los anuncios. Además, tanto España como Serbia tienen una producción de cerveza en el ámbito nacional, y en ambos países encontramos grandes marcas de cerveza de cobertura nacional, aparte de la diversificación del sector en la producción a una escala más reducida, o de la importación de la cerveza de otros países.

De cada una de las marcas mencionadas hemos seleccionado tres anuncios, lo que hace el total de los 18 anuncios de la muestra. El periodo que abarca nuestra muestra va desde abril de 2017 hasta septiembre de 2018. Nos hemos centrado solamente en anuncios que pertenecen a campañas nacionales tanto en España como en Serbia, por lo tanto, en nuestra muestra no se incluyen anuncios de campañas internacionales. Tampoco se incluyen en nuestra muestra los anuncios pertenecientes a las campañas relacionadas con el Campeonato Mundial de fútbol que tuvo lugar en el año 2018, ni de ninguna otra promoción especial.

Los anuncios que hemos analizado en nuestra muestra, y que han sido consultados en los canales oficiales de las marcas de cerveza de Youtube, se listan a continuación con sus fechas de publicación correspondientes: Mahou Cinco Estrellas - Un sabor muy grande (publicado el 7 de septiembre de 2018); Mahou Barrica - Revienta las reglas (publicado el 1 de marzo de 2018); Mahou - Maestra para Maestros - Servidores Online (publicado el 2 de octubre de 2017); Cruzcampo Radler - 35 grados a la sombra (publicado el 25 de julio de 2018); Cruzcampo Radler - Dicen que la piscina da hambre (publicado el 17 de julio de 2018); Cruzcampo - Libera lo que sientes (publicado el 25 de abril de 2018); Estrella Damm - Álex y Julia (publicado el 10 de junio de 2018); Estrella Damm - La receta (publicado el 7 de mayo de 2018); Estrella Damm - Mediterráneamente / Cyrano (publicado el 28 de marzo

de Heineken Srbija, Carlsberg Srbija y Molson Coors - mayores productores de cerveza de Serbia.

${ }^{5}$ En el caso de la marca Зајечарско (Zaječarsko), dado que su nombre oficial está en cirílico, en la primera mención usamos su nombre original Зајечарско, con su equivalente en el alfabeto serbio latino Zaječarsko. En las menciones posteriores, usamos el equivalente en el alfabeto latino. 
de 2018, nueva publicación el 3 de marzo de 2019); Jelen - Pun ukus života (publicado el 22 de febrero de 2018); Jelen - Naša stvar (publicado el 26 de diciembre de 2017); Jelen - Zov prijatelja (publicado el 27 de abril de 2017); Lav - Velike stvari (publicado el 13 de abril de 2018); Lav - Od Lava za Lava (publicado el 23 de octubre de 2017); Lav - Ponosan na svoje tragove (publicado el 4 de julio de 2017); Zaječarsko - Pšenično je Zaječarsko (publicado el 26 de abril de 2018); Zaječarsko - Crno je Zaječarsko (publicado el 30 de octubre de 2017) y Zaječarsko - Svadba (publicado el 13 de abril de 2017).

\section{Resultados del análisis}

En primer lugar presentaremos los datos del análisis sociolingüístico que incluye las variables lingüísticas, los pronombres personales (véase Cuadro 1). Como podemos observar en el Cuadro 1, en términos totales del corpus, la presencia de la segunda persona del singular 'tú' supone un $50 \%$, seguida de la primera persona del plural 'nosotros', con un 27,8\%. Es bien sabida y estudiada esta característica del sociolecto publicitario de utilizar estas formas de los pronombres con el propósito de incluir al receptor en el mensaje y hacer el mensaje más cercano ${ }^{6}$.

\begin{tabular}{|c|c|c|c|c|}
\hline N.o de anuncios & $\begin{array}{c}\text { 2ª persona del } \\
\text { singular (TÚ) }\end{array}$ & $\begin{array}{c}\text { 1- } \text { persona del plural } \\
\text { (NOSOTROS) }\end{array}$ & Otros & Total \\
\hline España & 5 & 1 & 3 & 9 \\
\hline Serbia & 4 & 4 & 1 & 9 \\
\hline Total & 9 & 5 & 4 & 18 \\
\hline$\%$ & $50 \%$ & 27,8 & 22,2 & 100 \\
\hline
\end{tabular}

Cuadro 1. Pronombres personales en los anuncios de la muestra

Los datos de la columna 'Otros' recogen tendencias menos frecuentes, concretamente, en el caso de los anuncios de España se trata del uso de la primera persona del singular y de la segunda persona del plural, mientras que en el caso de Serbia tenemos un anuncio en el que se utiliza también la primera persona del singular.

\footnotetext{
${ }^{6}$ Tanto para el uso de pronombres personales como para una compilación de estudios sobre el sociolecto publicitario desde diferentes perspectivas teóricas y metodológicas véase L. Valozic (2015: 17-59).
} 
Como mencionamos anteriormente, también introducimos en nuestro análisis una dimensión que sobrepasaría los límites estrictos de la sociolingüística, ya que nos centramos en la imagen de los anuncios con el propósito de observar de qué manera se personifican los usos de la lengua ${ }^{7}$. En el siguiente cuadro (Cuadro 2) podemos ver qué se representa en la imagen del anuncio cuando se usan los pronombres personales.

\begin{tabular}{|c|c|c|c|c|c|c|}
\hline $\begin{array}{c}\text { N. }{ }^{\text {ande }} \text { de } \\
\text { ancios }\end{array}$ & $\begin{array}{c}\text { TÚ } \\
\text { (CONSUMI- } \\
\text { DOR) }\end{array}$ & $\begin{array}{c}\text { NOSO- } \\
\text { TROS } \\
\text { (CONSU- } \\
\text { MIDOR) }\end{array}$ & $\begin{array}{c}\text { NOSOTROS } \\
\text { (EMPRESA } \\
\text { CERVE- } \\
\text { RA) }\end{array}$ & $\begin{array}{c}\text { NOSOTROS } \\
\text { (EMPRESA } \\
\text { CERVECERA = } \\
\text { CONSUMI- } \\
\text { DOR) }\end{array}$ & Otros & Total \\
\hline España & 5 & 0 & 1 & 1 & 2 & 9 \\
\hline Serbia & 4 & 2 & 1 & 1 & 1 & 9 \\
\hline Total & 9 & 2 & 2 & 2 & 3 & 18 \\
\hline$\%$ & $50 \%$ & 11,1 & 11,1 & 11,1 & 16,7 & 100 \\
\hline
\end{tabular}

Cuadro 2. Referencia en imagen del pronombre en los anuncios de la muestra

La forma predominante en todo el corpus 'tú' se representa en la imagen de los anuncios a través de diferentes personajes, diferentes perfiles de personas a las que se refiere ese 'tú', y así se identifica el tipo de consumidor al que van dirigidos los anuncios.

Otra característica común en los anuncios de ambos países es el uso de un 'nosotros' inclusivo que se refiere, o bien a la empresa cervecera, o bien a la empresa cervecera y al consumidor. Esta también es una característica ampliamente observada en diferentes tipos de anuncios, donde el 'nosotros' se puede referir o a la empresa, o incluir al consumidor en una 'comunidad abstracta' liderada por una marca, en el sentido de crear un universo constituido por 'nosotros que creamos este producto y tú que lo consumes'.

Podemos ver también en el Cuadro 2 que solamente en los anuncios de Serbia tenemos una referencia exclusiva al consumidor con la segunda persona del plural, esto es, con el uso de 'nosotros'. Esto nos parece significativo, porque ese 'nosotros' está claramente personificado en la imagen a través de la figura de hombres de 25 a 55 años de edad, y es

\footnotetext{
${ }^{7}$ La disciplina que más se ha ocupado del estudio de la imagen en el sociolecto publicitario es la semiótica. En este ámbito de estudio cabe mencionar trabajos de R. Barthes (2001), E. Feliu (1984) y G. Péninou (1976), entre otros (Valozic 2015: 21-28).
} 
inclusivo en la medida en que el consumidor se pueda sentir identificado con dicha personificación del 'nosotros', y no inclusivo si el consumidor no se identifica con los personajes que protagonizan el anuncio. Aquí vemos una primera diferencia, donde en los anuncios de Serbia hay una clara definición de ese 'nosotros' consumidores de cerveza, mientras que en los anuncios españoles de nuestra muestra no encontramos esta manera de definir al consumidor de cerveza.

Aparte de la diferencia anteriormente mencionada, también encontramos otra diferencia, en este caso relacionada con el uso del pronombre 'vosotros' en los anuncios de España (Cuadro 3). Se trata de un 'vosotros' que se refiere en ambos anuncios a los jóvenes. Cabe destacar, también, que estos dos anuncios que hacen referencia a los jóvenes, establecen también un paralelismo entre referencias culturales más tradicionales y referencias culturales de actualidad.

\begin{tabular}{|c|c|c|c|}
\hline N.. anuncios & $\begin{array}{c}\text { VOSOTROS } \\
\text { (CONSUMIDORES) }\end{array}$ & Otros & Total \\
\hline España & 2 & 7 & 9 \\
\hline Serbia & 0 & 9 & 9 \\
\hline Total & 2 & 16 & 18 \\
\hline
\end{tabular}

Cuadro 3.

Referencia en imagen del pronombre 'vosotros' en los anuncios de España

Continuando con el análisis ampliado, nos enfocamos en nuestra variable social, los anuncios de cerveza, y dentro de los anuncios, en la imagen, observamos las variables de sexo y edad de los diferentes personajes. Al observar la distribución de las variables de sexo y edad en los anuncios de España, en el Cuadro 4 vemos que tanto los hombres como las mujeres están reflejados en los anuncios. Tenemos un porcentaje menor, de $22,2 \%$ del total, donde los protagonistas son hombres en torno a los 30 años de edad, y un restante $77,8 \%$ de anuncios donde las mujeres aparecen como protagonistas, coprotagonistas o como personajes secundarios. 


\begin{tabular}{|c|c|c|c|c|c|}
\hline & $\begin{array}{c}\text { Protago- } \\
\text { nista } \\
\text { Hombre / } \\
30 \text { años }\end{array}$ & $\begin{array}{c}\text { Protago- } \\
\text { nista } \\
\text { Mujer / } \\
30 \text { años }\end{array}$ & $\begin{array}{c}\text { Protagonista } \\
\text { Hombre } 30-45+ \\
\text { años } \\
\text { Secundarios } \\
\text { H / M / 30 años }\end{array}$ & $\begin{array}{c}\text { Protagonis- } \\
\text { tas } \\
\text { Hombre / } \\
\text { Mujer } \\
25-50+ \\
\text { años }\end{array}$ & Total \\
\hline España & 2 & 1 & 2 & 4 & 9 \\
\hline$\%$ & 22,2 & \multicolumn{3}{|c|}{77,8} & 100 \\
\hline
\end{tabular}

Cuadro 4. Variables de sexo y edad de los personajes en imagen - España

Los datos porcentuales del Cuadro 5 nos indican una diferencia con respecto a España, ya que el porcentaje de anuncios donde el hombre es el protagonista es de un 44,4\%, mientras que no tenemos anuncios serbios en la muestra donde la mujer es protagonista única, es decir, la consumidora meta del anuncio. Sin embrago, las mujeres aparecen como protagonistas junto con los hombres en un $22,2 \%$ de los anuncios. Al sumar las columnas donde las mujeres aparecen como protagonistas junto con los hombres, con la columna donde aparecen como personajes secundarios, obtenemos un porcentaje mayor, un $44,4 \%$, pero todavía distante del porcentaje que hemos visto en los anuncios españoles, un 77,8\%.

\begin{tabular}{|c|c|c|c|c|c|c|}
\hline & $\begin{array}{c}\text { Protago- } \\
\text { nista } \\
\text { Hombre } \\
25-50 \\
\text { años }\end{array}$ & $\begin{array}{c}\text { Protago- } \\
\text { nistas } \\
\mathrm{H} / 45+ \\
\text { años } \\
\text { M / } 30 \\
\text { años }\end{array}$ & $\begin{array}{c}\text { Protagonis- } \\
\text { tas } \\
\mathrm{H} / 30-45+ \\
\text { años } \\
\text { años } \\
\text { añ }\end{array}$ & $\begin{array}{c}\text { Protago- } \\
\text { nista } \\
\text { Cerveza } \\
\text { Secunda- } \\
\text { rios } \\
\text { H / M / 30 } \\
\text { años }\end{array}$ & $\begin{array}{c}\text { Prota- } \\
\text { gonista } \\
\text { Cerveza }\end{array}$ & Total \\
\hline Serbia & 4 & 1 & 1 & 2 & 1 & 9 \\
\hline$\%$ & 44,4 & \multicolumn{2}{|c|}{22,2} & 22,2 & 11,1 & 100 \\
\hline
\end{tabular}

Cuadro 5. Variables de sexo y edad de los personajes en imagen - Serbia

Podemos concluir así que, en términos generales, vemos un reflejo de las diferencias culturales y sociales de los dos países en los anuncios que conforman nuestra muestra de trabajo. 


\section{Conclusiones}

En este análisis comparativo de los anuncios de cerveza de España y Serbia que conforman nuestra muestra, podemos observar claramente que el sociolecto publicitario refleja las diferencias culturales del contexto social y cultural en el que se desarrolla. Estas diferencias se observan en la actualidad, y coinciden con el anteriormente mencionado proceso social, que convive con la globalización económica, en el que perdura la aspiración a la diversificación mediante la reivindicación de elementos autóctonos sociales, culturales y lingüísticos. Sin embargo, también se perciben algunas tendencias presentes en el ámbito mundial, vinculadas a la globalización económica, que estarían en sintonía con la tendencia de uniformar el ámbito financiero, político, cultural y social de los países del mundo.

El sociolecto publicitario que hemos analizado presenta, pues estas tendencias más globales de la publicidad del uso del pronombre 'tú', que incluye al consumidor y se acerca a él. También está presente el uso del 'nosotros', donde la empresa o la marca incluyen al consumidor dentro de su universo, al consumir cierto producto, el consumidor es parte del universo que crea la marca o el producto.

Por otra parte, hemos observado ciertas diferencias, donde los anuncios de cerveza de Serbia utilizan la estrategia del 'nosotros', que define muy bien al consumidor como hombre de entre 25 y 55 años de edad y a su vez, sin expresarlo explícitamente, no incluye a otros consumidores. Otra diferencia importante está en los anuncios de marcas españolas que utilizan un 'vosotros' para referirse en imagen a los jóvenes, y a la vez hacen que convivan las referencias culturales más tradicionales con las contemporáneas.

Asimismo, se observa la diferencia en el hecho de que en los anuncios serbios, la mujer no es protagonista directa de los anuncios de cerveza, y en los anuncios de España sí. Además, en los anuncios españoles, las mujeres aparecen como protagonistas o coprotagonistas en un $77,8 \%$ de los anuncios analizados, mientras que en los anuncios serbios aparecen solo como coprotagonistas o personajes secundarios en un $44,4 \%$.

Cabe destacar un dato anecdótico, aunque aun así ilustrativo, de la muestra de trabajo. Concretamente, en nuestra muestra tenemos un anuncio de España y un anuncio de Serbia donde los protagonistas son padre e hija, por lo tanto, coinciden en cuanto al tema principal. Sin embargo, en el anuncio español, padre e hija están sentados en un 
bar, que, como se explica en el anuncio, hace años era una sala de cine $x$, toman cerveza juntos, $\mathrm{y}$ el anuncio gira en torno a la convivencia de 'los valores de antes', representados por el padre, y 'los valores de hoy', representados por la hija. En el anuncio serbio se presenta al padre y a la hija en la boda de la hija, donde el padre, que también representa 'los valores de antes', abraza a su hija el día de su boda, y solo se muestran en el anuncio alusiones tradicionales a la ceremonia de celebración de un nuevo matrimonio. Vemos así, que en el uso de un mismo tema, en este caso padre e hija, incluso si miramos la figura del padre como una representación de referencias culturales más tradicionales y la figura de la hija como una representación de referencias culturales contemporáneas, la interpretación y el desarrollo de la historia son bien diferentes.

Por lo tanto, podemos concluir que la diversidad cultural está presente en el sociolecto publicitario contemporáneo, y su análisis así lo evidencia, aunque, también refleja la otra vertiente del mundo contemporáneo más orientada a la globalización.

La publicidad, por supuesto, no refleja toda la complejidad del contexto social y cultural en el que se desarrolla, pero sí recoge algunos de sus elementos. Consideramos, pues, como también lo han destacado otros investigadores, que esta cualidad de la publicidad la hace útil para su análisis en clase (y particularmente en clase de ELE), especialmente por su carácter ilustrativo, ya que al comparar anuncios de diferentes países podemos observar muestras de la diversidad cultural.

\section{BIBLIOGRAFÍA}

Barthes, Roland. La Torre Eiffel: textos sobre la imagen. Barcelona: Paidós Ibérica, 2001. Impreso.

Durán Martínez, Ramiro. "Covert communication in the promotion of alcohol and tobacco in spanish press advertisements". RAEL, 4 (2005): 82-102. Web. 07 Jul. 2018.

Eguizábal, Raúl. Historia de la publicidad. Madrid: Eresma y Celeste Ediciones, 1998. Impreso.

Feliu, Emilio. Los lenguajes de la publicidad. Alicante: Secretaría de publicaciones de la Universidad de Alicante, 1984. Impreso.

Gimeno, Francisco. Dialectología y sociolingüística españolas. $2^{\underline{a}}$ ed. Alicante: Universidad de Alicante, 1990. Impreso.

Gimeno, Francisco \& María Victoria Gimeno. El desplazamiento lingüístico del español por el inglés. Madrid: Cátedra, 2003. Impreso. 
Péninou, Georges. Semiótica de la publicidad. Barcelona: Gustavo Gil, 1976. Impreso.

Racionero Siles, Flora \& María Ángeles Olivares García. "Estudio comparativo de los estereotipos femeninos en la publicidad gráfica de España y Brasil, a partir de una experiencia educativa en la enseñanza superior". Revista Iberoamericana de Educación, Vol. 59, Núm. 4 (2012): S. P. Web. 01 Jul. 2018.

Ramos Serrano, Marina \& María del Mar Rubio Hernández. "La identidad cultural en la publicidad de bebidas alcohólicas". Pensar la Publicidad, Vol. 5, Núm. 2 (2011): 205-231. Web. 30 Sep. 2018.

Romero Gualda, María Victoria. El español en los medios de comunicación. Madrid: Arco Libros, 1993. Impreso.

Valozic, Luiza. El anglicismo léxico en la publicidad. Sant Vicent del Raspeig: Publicacions de la Universitat d'Alacant. 2015. Impreso.

\section{CULTURAL DIVERSITY AND ADVERTISING: A COMPARATIVE ANALYSIS OF BEER ADVERTISEMENTS IN SPAIN AND SERBIA}

\section{Summary}

The relevance of the mass media and advertising for the society has been increasing since the second half of the 20th century and today it is probably more important than ever. Both the mass media and advertising have been studied by many different theoretical and methodological perspectives and still today there is a continued interest amongst researchers in their study. Due to its importance within society and its influence therein, many linguistic disciplines have studied advertising, especially due to the potential it has to influence the use of language and to transmit the tendencies related to globalisation.

Our paper presents the results of a theoretical and methodological sociolinguistic comparative analysis of beer advertisements in Spain and Serbia. In addition, we also present an extended analysis of our social variable (beer advertisements in Spain and Serbia), which is not strictly sociolinguistic, and within the extended analysis we also comment on the image of the advertisements with the objective of analysing the representation of the advertisement message recipients.

The objective of our comparative study is to observe the cultural and social diversity present in the advertisements of both countries and also to stress the usefulness of comparative analysis of advertising in teaching Spanish as a foreign language.

Keywords: cultural diversity, advertising, globalisation, sociolinguistic analysis. 